\title{
Cronograma de Observaciones Solares para el sensor SIS en la misión ExoMars16
}

\author{
Gonzalo BARDERAS ${ }^{1}$, Sandra GOICOECHEA ${ }^{1}$ y Pilar ROMERO ${ }^{1,2}$ \\ ${ }^{1}$ S.D. Astronomía y Geodesia ${ }^{2}$ Instituto de Matemática Interdisciplinar \\ Facultad de Matemáticas/Universidad Complutense \\ gonzalobm@mat.ucm.es; sandragoicoechearuiz@gmail.com; pilar@ucm.es
}

Recibido: 09/05/2016

Aceptado: 20/06/2016

\begin{abstract}
Resumen
La Misión ExoMars16 es la primera misión del programa ExoMars de la ESA, cuyo lanzamiento se realizó el día 14 de marzo de 2016 estando prevista su llegada a Marte en octubre de 2016. Esta misión consta del módulo orbital Gas Tracer Orbiter y del módulo de descenso Schiaparelli. Este último está equipado con tecnología experimental entre la que se encuentra el Sensor de Irradiación Solar (SIS) desarrollado por el Instituto Nacional de Técnica Aeroespacial, incluido dentro del experimento para la caracterización del entorno atmosférico sobre la superficie marciana DREAMS. El principal objetivo del sensor SIS es la medición del espectro solar en la superficie de Marte, la transparencia atmosférica y la concentración de polvo para, combinado con los datos del sensor de campos eléctricos MicroARES, estudiar los mecanismos que inician las tormentas de polvo marcianas.

El objetivo de este trabajo es caracterizar la visibilidad del Sol desde el módulo Schiaparelli y establecer un cronograma de observaciones solares para SIS en el tiempo de vida previsto para el módulo sobre la superficie de Marte de 2 a 8 soles. Para establecer el cronograma de observaciones solares se ha calculado la posición del Sol vista desde SIS, determinando el TU de los ortos y ocasos, así como el origen del Local True Solar Time (LTST) en el lugar de amartizaje. Para el cálculo de las coordenadas locales, azimut y altura, se ha realizado una simulación de Montecarlo de 1000 posiciones aleatorias dentro de la elipse de amartizaje prevista por la ESA. Por último, se establece el cronograma de visibilidad del Sol más probable, así como para los puntos característicos de la elipse, que acotan los márgenes de error de dicha determinación. Esto permite planificar adecuadamente las observaciones de radiación solar con SIS. El Sol estará visible durante aproximadamente 12 horas y 26 minutos cada sol.
\end{abstract}

Palabras clave: SIS; EXOMARS; observaciones de radiación solar.

\section{Chronogram of Solar Observations for the EXOMARS16 Solar Irradiance}

\section{Sensor}

\begin{abstract}
ExoMars16 is the first mission of the ESA ExoMars programme. Its launch is planned for the Mars 2016 window and the arrival to Mars in October 2016. The mission consists of the Trace Gas Orbiter and an entry, descent and landing demonstrator module known as Schiaparelli. The latter is equipped with new testing technologies; including a Solar Irradiation Sensor (SIS) developed by the Instituto Nacional de Técnica Aeroespacial. SIS belongs to the DREAMS experiment to characterize the atmospheric environment of the Mars surface. SIS main goal is to measure the solar spectrum in Mars, the transparency of
\end{abstract}


the atmosphere and the atmospheric dust content. Combined with the measurement of the electric fields from MicroAres, these measurements will allow to study the role of electric forces on dust lifting, the mechanism that initiates dust storms.

The objective of this work is to characterize the visibility of the Sun from the Schiaparelli module and to establish a chronogram of solar observations for SIS. For this establishment the Sun position as seen from SIS has been calculated, determining the sunrises and sunsets times in UT. To obtain local coordinates, azimuth and elevation, a Montecarlo simulation of 1000 random positions within the ESA forecasted landing ellipse for Schiaparelli. Then, the most probable Sun visibility chronogram is determined, as well as the chronogram for the ellipse characteristic points that limit the error margins of this determination. This allows planning adequately the solar irradiation observations for SIS. The Sun will be visible for approximately 12 hours and 26 minutes each sol.

Key words: SIS; EXOMARS; solar radiation observations.

Sumario: Introducción. 1. Determinación de las coordenadas del Sol 1.1 Coordenadas areocéntricas areoecuatoriales 1.2. Azimut y altura. 2. Elipse de amartizaje de Schiaparelli. 3. Cronograma. 4. Conclusiones. Agradecimientos. Referencias.

\section{Referencia normalizada}

Barderas, G. et al. (2016). Cronograma de Observaciones Solares para el sensor SIS en la misión ExoMars16. Física de la Tierra, Vol., 28, 83-95.

\section{Introducción}

Marte es uno de los objetivos prioritarios de la exploración espacial de la presente y próximas décadas, en las que está previsto el envío de diversas misiones de diferentes agencias espaciales. La Misión ExoMars 16 es la primera del programa ExoMars de la ESA. Su lanzamiento se realizó el 14 de marzo de 2016, estando prevista su llegada a Marte en octubre de 2016. Consta de un módulo orbital, el Trace Gas Orbiter, y del módulo Schiaparelli de entrada, descenso y aterrizaje (Vago, 2014). Los principales objetivos de ExoMars16 son encontrar evidencias de metano y otros gases atmosféricos que pudieran ser signos de procesos geológicos o posible actividad biológica, además de poner a prueba tecnologías en fase de prueba para la contribución de la ESA en posteriores misiones a Marte (ESA, 2016).

El módulo Schiaparelli está equipado con tecnología experimental, que incluyen entre otros: material especial de protección térmica, un sistema de paracaídas, un radar Doppler y un sistema de frenado controlado por propulsión líquida. Debido a la limitada capacidad de sus baterías, el módulo estará operativo aproximadamente entre 2 y 8 soles (días marcianos, de $24^{\mathrm{h}} 39^{\mathrm{m}} 35.244^{\mathrm{s}}$ (Folkner et al., 2008)) sobre la superficie de Marte.

El objetivo de este trabajo es caracterizar la visibilidad del Sol para el módulo Schiaparelli de la misión ExoMars 16 y establecer un cronograma de observaciones solares previo al lanzamiento de la misión para programar los tiempos de observación del Sensor de Irradiancia Solar (SIS, Álvarez, 2014) que forma parte del experimento DREAMS dentro del módulo Schiaparelli (Bettanini et al., 2014). El objetivo principal del SIS, desarrollado por el Instituto Nacional de Técnica Aeroespacial (INTA), 
es la medición del espectro solar y sus variaciones a lo largo del día y la estimación de la profundidad óptica atmosférica y la concentración de polvo para, entre otros objetivos estudiar conjuntamente con la instrumentación de DREAMS los mecanismos que desencadenan las tormentas de polvo (Silvestro, 2014).

Para el establecimiento de este cronograma de observaciones solares se calculan en primer lugar la posición del Sol vista desde SIS en un sistema de coordenadas horizontales (azimut y altura). El procedimiento empleado se describe en la sección 1. En la sección 2 se detalla el procedimiento para simular las posiciones aleatorias del observador dentro de la elipse de amartizaje para Schiaparelli desde las que se realiza la simulación de Montecarlo del cálculo del azimut y la altura del Sol. En la sección 3 se presentan los resultados y establecen los cronogramas de observación que permitirán planificar las observaciones de la radiación solar con el sensor SIS y el análisis futuro de dichas observaciones. Finalmente, las principales conclusiones se resumen en la sección 4.

\section{Determinación de las coordenadas del Sol}

Las coordenadas horizontales del centro del Sol, azimut, $A_{s}$, y altura sobre el horizonte, $h_{s}$, para un observador de coordenadas conocidas, latitud, $\varphi$, y longitud, $\lambda$, sobre la superficie de Marte, se han determinado a partir de las coordenadas areoecuatoriales areocéntricas del Sol. Estas coordenadas se han derivado de los elementos keplerianos clásicos de Marte y de sus tasas de cambio referidos a la eclíptica y equinoccio de la época J2000.0, utilizadas para la planificación de observaciones y el diseño de misiones e instrumentación espacial (Romero et al., 2011). Los valores empleados para los elementos orbitales (ver Fig. 1) $a$, semieje mayor de la órbita, $e$ excentricidad, $i$ inclinación sobre la eclíptica, $\Omega$ longitud del nodo ascendente, $\varpi$ longitud del perihelio, se listan en la Tabla 1 (Standish y Williams, 2009). Se describen a continuación los procedimientos seguidos para la obtención de las coordenadas areocéntricas areoecuatoriales del centro del Sol y su transformación a coordenadas horizontales. 


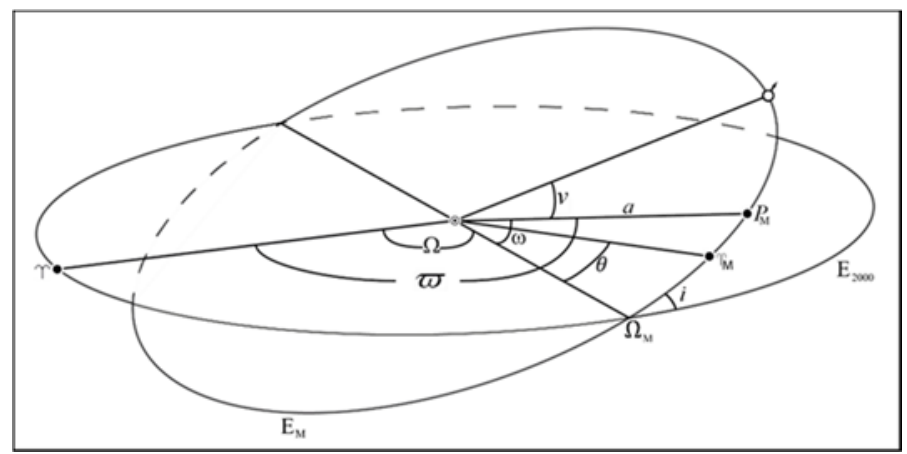

Figura 1. Elementos orbitales, referidos a la eclíptica, E2000, y equinoccio, $\Upsilon$, de la época J2000.

\subsection{Coordenadas areocéntricas areoecuatoriales}

En primer lugar, se considera un sistema de referencia areocéntrico $\{M ; x, y, z\}$ con plano fundamental $x y$ el plano de la órbita media de Marte y dirección del eje $x$, el equinoccio medio de la fecha. En este sistema de referencia las coordenadas del Sol pueden expresarse, como función de la posición de Marte, en términos del radio vector de Marte, $d$, de su anomalía verdadera medida desde el perihelio, $v$, y del argumento del perihelio, $\omega=\varpi-\Omega$ (Montenbruck y Gill, 2000), como:

$$
\left(\begin{array}{l}
x \\
y \\
z
\end{array}\right)=-\left(\begin{array}{c}
d \cos (v+\omega-\theta) \\
d \operatorname{sen}(v+\omega-\theta) \\
0
\end{array}\right),(1)
$$

donde $\theta$ es la longitud de arco $\left(\Omega_{M} \Upsilon_{M}\right)$, medido a lo largo de la órbita de Marte desde el nodo ascendente de la órbita de Marte sobre la eclíptica hasta el equinoccio de Marte, $\Upsilon_{M}$ (ver Fig. 1).

La transformación desde este sistema de referencia a un sistema de referencia areocéntrico areoecuatorial $\{M ; X, Y, Z\}$ con plano fundamental, $X Y$, el ecuador de Marte, eje $X$ en la dirección del meridiano origen de longitudes, Airy- 0 , eje $Z$ en la dirección del polo de rotación e $Y$ completando un triedro directo puede realizarse mediante la composición de dos rotaciones elementales. Una primera rotación, alrededor del eje $x$, de ángulo la oblicuidad del ecuador de Marte sobre la órbita de Marte, $\varepsilon$, y una segunda rotación, alrededor del eje $Z$, de ángulo $\left(V_{M}+\lambda\right)$, con $V_{M}$ la longitud del equinoccio de Marte medido sobre el ecuador desde Airy-0 y $\lambda$ la longitud geográfica del observador. En la Fig. 2 se describen los sistemas de referencia y los ángulos de orientación involucrados en esta transformación (Barderas et al., 2012), 
cuyos valores se listan en la Tabla 1. De esta forma la transformación, en notación matricial, se escribe:

$$
\left(\begin{array}{l}
X \\
Y \\
Z
\end{array}\right)=R_{Z}\left(V_{M}+\lambda\right) R_{x}(\varepsilon)\left(\begin{array}{l}
x \\
y \\
z
\end{array}\right) .
$$

\subsection{Azimut y altura}

La transformación final a un sistema de referencia local sobre la superficie de Marte $\{\mathrm{O} ; \xi, \rho, \zeta\}$, con origen el observador $\mathrm{O}$, de coordenadas $(\varphi, \lambda)$, plano fundamental $(\xi \rho)$ el horizonte local, y ejes, $\xi$ en la dirección del Norte, $\zeta$ en la dirección del zénit y $\rho$ formando triedro retrógrado, puede efectuarse mediante dos rotaciones adicionales. Una primera alrededor del eje $Y$, de ángulo $\left(90^{\circ}-\varphi\right)$; y una segunda alrededor del eje $\zeta$, de ángulo $180^{\circ}$, para medir azimuts desde el Norte. Esto es:

$$
\left(\begin{array}{l}
\xi \\
\rho \\
\zeta
\end{array}\right)=R_{\xi}\left(180^{\circ}\right) \mathrm{R}_{Y}\left(90^{\circ}-\phi\right)\left(\begin{array}{c}
X \\
Y \\
Z
\end{array}\right)
$$

Finalmente, el azimut As y la altura $h$ s del centro del sol se determinan a partir de las correspondientes coordenadas cartesianas horizontales $(\xi, \rho, \zeta)$ mediante:

Tabla 1. Elementos Orbitales de Marte y parámetros de orientación de sistemas de referencia

\begin{tabular}{|c|c|c|c|}
\hline \multicolumn{2}{|l|}{ Parámetro } & Valor & Variación \\
\hline Semieje mayor & $a^{1}$ (ua, ua/siglo) & 1.52371034 & 0.00001847 \\
\hline Excentricidad & $e^{1}(\mathrm{rad}, \mathrm{rad} / \mathrm{siglo})$ & 0.09339410 & 0.00001847 \\
\hline Inclinación & $i^{1}\left({ }^{\circ}, \%\right.$ siglo $)$ & 1.84969142 & -0.0081313 \\
\hline Longitud del periares & $\varpi^{1}\left({ }^{\circ},{ }^{o} /\right.$ siglo $)$ & -23.94362959 & 0.44441088 \\
\hline Longitud del nodo ascendente & $\Omega^{1}(\mathrm{o}, \mathrm{o} /$ siglo $)$ & 49.55953891 & -0.29257343 \\
\hline $\begin{array}{l}\text { Arco } \Omega_{M} \Upsilon_{M} \text { sobre la órbita de } \\
\text { Marte }\end{array}$ & $\theta^{1}(\mathrm{o}, \%$ día $)$ & 35.4371 & $-5.76 \times 10^{-6}$ \\
\hline $\begin{array}{l}\text { Oblicuidad de la órbita de } \\
\text { Marte }\end{array}$ & $\varepsilon^{1}\left({ }^{o}\right.$, o $/$ día $)$ & 25.1886 & $-1.094 \times 10^{-7}$ \\
\hline $\begin{array}{l}\text { Arco } \Upsilon_{M} \eta_{M} \text { sobre el ecuador de } \\
\text { Marte }\end{array}$ & $V_{M}^{2}\left({ }^{o}, \% /\right.$ día $)$ & 313.3828 & 350.89198226 \\
\hline
\end{tabular}
Tierra-Marte. Valores ${ }^{1}$ según Standish y Williams (2009), y ${ }^{2}$ según Burkhart (2006). 


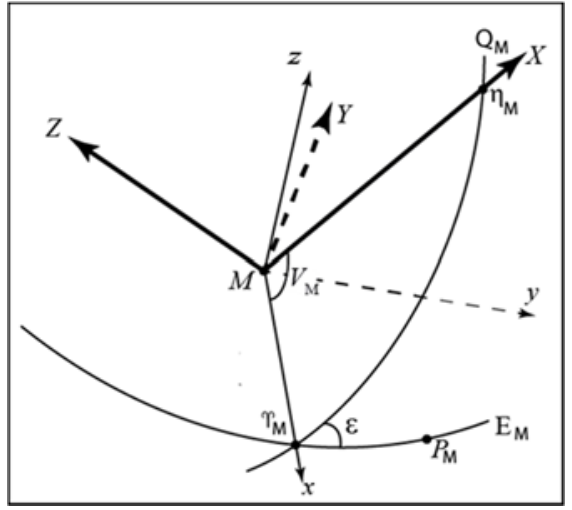

Figura 2. Sistemas de referencia utilizados en el cálculo de efemérides del Sol. $\{M ; x, y, z\}$ es el sistema de referencia areocéntrico con plano fundamental $x y$ el plano de la órbita media de Marte y dirección del eje $\mathrm{x}$, el equinoccio medio de Marte. $\{M ; X, Y, Z\}$ es el sistema de referencia areocéntrico areoecuatorial, con eje $X$ en la dirección del meridiano origen de longitudes, Airy-0. El punto $\mathrm{P}_{\mathrm{M}}$ se corresponde con la dirección del periares de la órbita, $\Upsilon_{M}$ con el equinoccio de Marte, $\eta_{M}$ con la intersección entre el meridiano origen y el ecuador de Marte, $Q_{\mathrm{M}} . V_{M}$ es el arco $\Upsilon_{M} \eta_{M}$ medido sobre el ecuador de Marte. $\varepsilon$ es la oblicuidad del ecuador de Marte sobre la órbita de Marte.

$$
\begin{aligned}
& A_{S}=\arctan \frac{\rho}{\xi}, \\
& h_{S}=\arctan \frac{\zeta}{\left(\xi^{2}+\rho^{2}\right)^{1 / 2}} .
\end{aligned}
$$

Los tiempos en TU correspondientes a los ortos y ocasos del Sol quedan determinados por la condición $h_{S}=0$. Las $0^{\mathrm{h}}$ de LTSL corresponden al TU para el valor máximo de $h_{S}$.

\section{Elipse de amartizaje de Schiaparelli}

El amartizaje del módulo Schiaparelli está previsto en un entorno del día 19 de octubre de 2016 en la planicie Meridiani Planum en las coordenadas $\left(\varphi_{0}, \lambda_{0}\right)=$ $\left(1^{\circ} .82 \mathrm{~S}, 353^{\circ} .85 \mathrm{E}\right)$, próximo a la zona de amartizaje del MER Opportunity (ver Fig. 3). El objetivo de la misión es alcanzar dicho punto con unas precisiones radial y transversal de $50 \mathrm{~km} \times 7.5 \mathrm{~km}$, si bien, debido a la incertidumbre existente en las fases de entrada, descenso y amartizaje, dichos límites de error se han extendido a una 
elipse de 100x30 km². El semieje mayor de dicha elipse de error tiene una orientación prevista entre $118^{\circ}$ y $125^{\circ}$ desde el Norte (Portigliotti, 2014).

Para el establecimiento del cronograma de observaciones solares para el sensor SIS y tener una cota de los errores cometidos asociados a la posición final real de amartizaje, se han analizado las variaciones en la posición del Sol para distintos puntos dentro de la elipse. Para este análisis se han elegido el centro de la elipse y los extremos de los semiejes, A, B, C, y D, que denominamos puntos característicos. Asimismo se ha tomado una muestra de 1000 puntos aleatorios en el interior de la elipse (Barderas et. al, 2012). Sus coordenadas se han determinado a partir de la generación de dos números aleatorios, $\mathrm{r}_{1}$ y $\mathrm{r}_{2}$, elegidos con una distribución uniforme en el intervalo $[0,1]$, de forma análoga a la descrita para el disco de radio unidad por Pharr y Humphreys (2004) mediante las ecuaciones:

$$
\begin{aligned}
& \varphi=\varphi_{0}+a \sqrt{r_{1}} \cos \left(2 \pi r_{2}\right) \sin \sigma+b \sqrt{r_{1}} \sin \left(2 \pi r_{2}\right) \cos \sigma, \\
& \lambda=\lambda_{0}+a \sqrt{r_{1}} \cos \left(2 \pi r_{2}\right) \cos \sigma-b \sqrt{r_{1}} \sin \left(2 \pi r_{2}\right) \sin \sigma,
\end{aligned}
$$

donde $a=50 \mathrm{~km}$ y $b=15 \mathrm{~km}$ son los semiejes mayor y menor respectivamente. El ángulo $\sigma$ se corresponde con el ángulo de orientación de la elipse respecto del eje longitudinal, fijado en $120^{\circ}$. En la Fig. 4 aparecen los puntos para los cuales se han realizado las determinaciones de azimut y altura y de los instantes de tiempo en TU para ortos, ocasos y pasos por el meridiano.

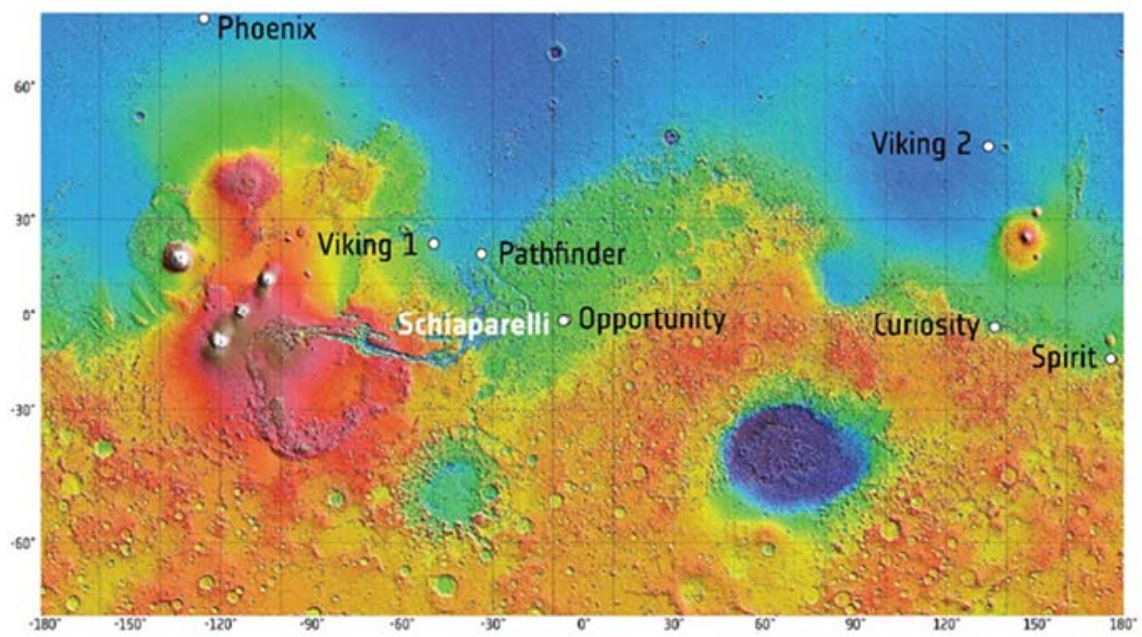

Figura 3. Lugar de amartizaje previsto para el módulo Schiaparelli. Se muestra conjuntamente con todos los landers enviados a Marte hasta la fecha. Copyright: mapa de la ESA; imagen de fondo MOLA Science Team. 


\section{Cronograma}

A continuación se analizan los resultados obtenidos utilizando en las fórmulas (1), (2), (3) y (4) de la sección 1 las coordenadas $(\varphi, \lambda)$ de los puntos característicos y de los puntos interiores de la elipse calculados mediante (5). En la Fig. 5 se muestran para 4 soles (que se corresponde a las fechas 18-21 de octubre de 2016) las variaciones de azimut y altura del Sol para el centro de la elipse y los puntos característicos A, B, C y D, respectivamente. Puede comprobarse cómo el azimut cubre únicamente un rango de $120^{\circ}$ del horizonte, aproximadamente entre $112^{\circ}$ y $247^{\circ}$. Esto se debe a que la culminación superior del Sol en este período se produce entre el zénit del observador y el Polo Sur ya que la declinación del Sol en estos días es de $-22^{\circ} .5$, siendo la latitud del observador de $1^{\circ} .82 \mathrm{~S}$. El tiempo que el Sol está por encima del horizonte es aproximadamente 12 horas y 26 minutos (Goicoechea, 2015).

La Tabla 2 resume los valores del azimut al orto y al ocaso del Sol y las horas en TU obtenidos para los diferentes días para los cinco puntos característicos, así como la media recortada al $80 \%$ resultado de la simulación Montecarlo efectuada. Las diferencias máximas de valores de azimut dependiendo del lugar de amartizaje pueden variar para un mismo sol, creciendo en un rango de valores entre $\left[0^{\circ} .0179\right.$, $\left.0^{\circ} .0217\right]$ para el orto, y decreciendo entre $\left[0^{\circ} .0212,0^{\circ} .0167\right]$ para el ocaso, durante los 4 soles estudiados. La máxima variación media de azimut entre dos soles es de $0^{\circ} .1297$ en el orto y de $-0^{\circ} .1282$ en el ocaso. Dependiendo de la latitud, el tiempo que de media está el Sol por encima del horizonte 12 horas 26 minutos, puede variar en 1 minuto. En los 4 soles, para un mismo lugar, la iluminación solar puede asimismo aumentar en 1 minuto.

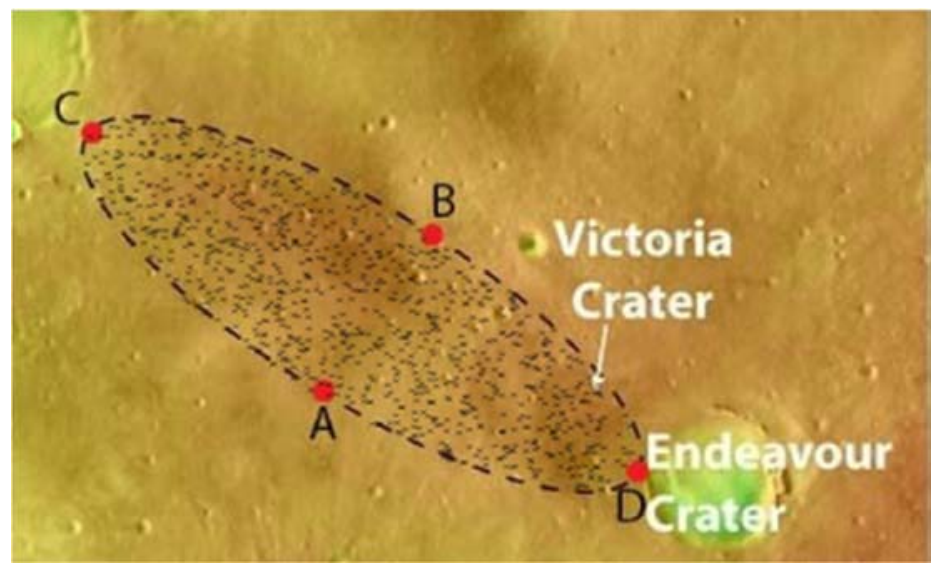

Figura 4. Elipse de amartizaje del módulo Schiaparelli mostrando los puntos característicos A, $\mathrm{B}, \mathrm{C}$ y D y las posiciones aleatorias utilizadas en las simulaciones. 
Tabla 2. Ortos y ocasos para 4 soles correspondientes a las fechas 18-21/oct/2016.

\begin{tabular}{crrrrrrr}
\hline \multirow{2}{*}{ Fecha } & Parámetro & $\begin{array}{c}\text { Media } \\
\text { Montecarlo }\end{array}$ & \multicolumn{1}{c}{ Centro } & \multicolumn{1}{c}{ A } & B & C & D \\
\hline \multirow{2}{*}{18} & Azimut Orto & 112.5259 & 112.5252 & 112.5243 & 112.5258 & 112.5182 & 112.5361 \\
Oct & Azimut Ocaso & 247.4071 & 247.4088 & 247.4080 & 247.4096 & 247.4136 & 247.3924 \\
16 & TU orto & $6: 38$ & $6: 38$ & $6: 39$ & $6: 37$ & $6: 41$ & $6: 35$ \\
& TU ocaso & $19: 04$ & $19: 04$ & $19: 05$ & $19: 03$ & $19: 05$ & $19: 04$ \\
19 & Azimut Orto & 112.6556 & 112.6563 & 112.6556 & 112.6569 & 112.6487 & 112.6679 \\
Oct & Azimut Ocaso & 247.2789 & 247.2825 & 247.2818 & 247.2831 & 247.2866 & 247.2668 \\
16 & TU orto & $7: 18$ & $7: 18$ & $7: 19$ & $7: 17$ & $7: 21$ & $7: 15$ \\
& TU ocaso & $19: 44$ & $19: 44$ & $19: 45$ & $19: 43$ & $19: 45$ & $19: 44$ \\
20 & Azimut Orto & 112.7822 & 112.7846 & 112.7839 & 112.7780 & 112.7763 & 112.7967 \\
Oct & Azimut Ocaso & 247.1537 & 247.1514 & 247.1503 & 247.1524 & 247.1625 & 247.1443 \\
16 & TU orto & $7: 58$ & $7: 58$ & $7: 59$ & $7: 58$ & $8: 01$ & $7: 55$ \\
& TU ocaso & $20: 25$ & $20: 25$ & $20: 26$ & $20: 24$ & $20: 25$ & $20: 24$ \\
21 & Azimut Orto & 112.9060 & 112.9099 & 112.9093 & 112.9031 & 112.9010 & 112.9227 \\
Oct & Azimut Ocaso & 247.0315 & 247.0311 & 247.0302 & 247.0320 & 247.0415 & 247.0248 \\
16 & TU orto & $8: 39$ & $8: 38$ & $8: 39$ & $8: 38$ & $8: 41$ & $8: 35$ \\
& TU ocaso & $21: 05$ & $21: 05$ & $21: 06$ & $21: 04$ & $21: 05$ & $21: 04$ \\
\hline
\end{tabular}

Tabla 3. Valores máximos de la altura en los puntos característicos de la elipse.

\begin{tabular}{lrrrrrr}
\hline Fecha & Parámetro & Centro & \multicolumn{1}{c}{ A } & \multicolumn{1}{c}{$\mathrm{B}$} & \multicolumn{1}{c}{$\mathrm{C}$} & \multicolumn{1}{c}{$\mathrm{D}$} \\
\hline \multirow{2}{*}{ 18/Oct/16 } & Altura máxima & 69.2725 & 69.3989 & 69.1460 & 68.5417 & 70.0031 \\
& TU & $12: 51$ & $12: 52$ & $12: 50$ & $12: 53$ & $12: 49$ \\
19/Oct/16 & Altura máxima & 69.1436 & 69.2701 & 78.3057 & 68.4114 & 69.8740 \\
& TU & $13: 31$ & $13: 32$ & $13: 30$ & $13: 33$ & $13: 29$ \\
\multirow{2}{*}{ 20/Oct/16 } & Altura máxima & 69.0176 & 69.1441 & 68.8910 & 68.2871 & 69.7483 \\
& TU & $14: 11$ & $14: 12$ & $14: 10$ & $14: 13$ & $14: 09$ \\
\multirow{2}{*}{ 21/Oct/16 } & Altura máxima & 68.8947 & 69.0210 & 68.7683 & 68.1641 & 69.6255 \\
& TU & $14: 51$ & $14: 52$ & $14: 50$ & $14: 53$ & $14: 49$ \\
\hline
\end{tabular}

Las diferencias en las horas para el TU al orto y al ocaso en días consecutivos se deben a la distinta duración de 1 sol y 1 día terrestre. Debido a posibles incertidumbres en la localización de los Landers sobre la superficie de Marte con respecto al meridiano origen de longitudes, los archivos de datos para ciertas aplicaciones específicas se almacenan como función de su LTST. Para facilitar la conversión entre LTST y TU se ha determinado la correspondencia de ceros entre las dos escalas, calculando el TU correspondiente a las $0^{\mathrm{h}}$ de LTST (respecto al meridiano superior), cuyos valores se muestran en la Tabla 3, junto con la altura del Sol correspondiente a la culminación superior. 

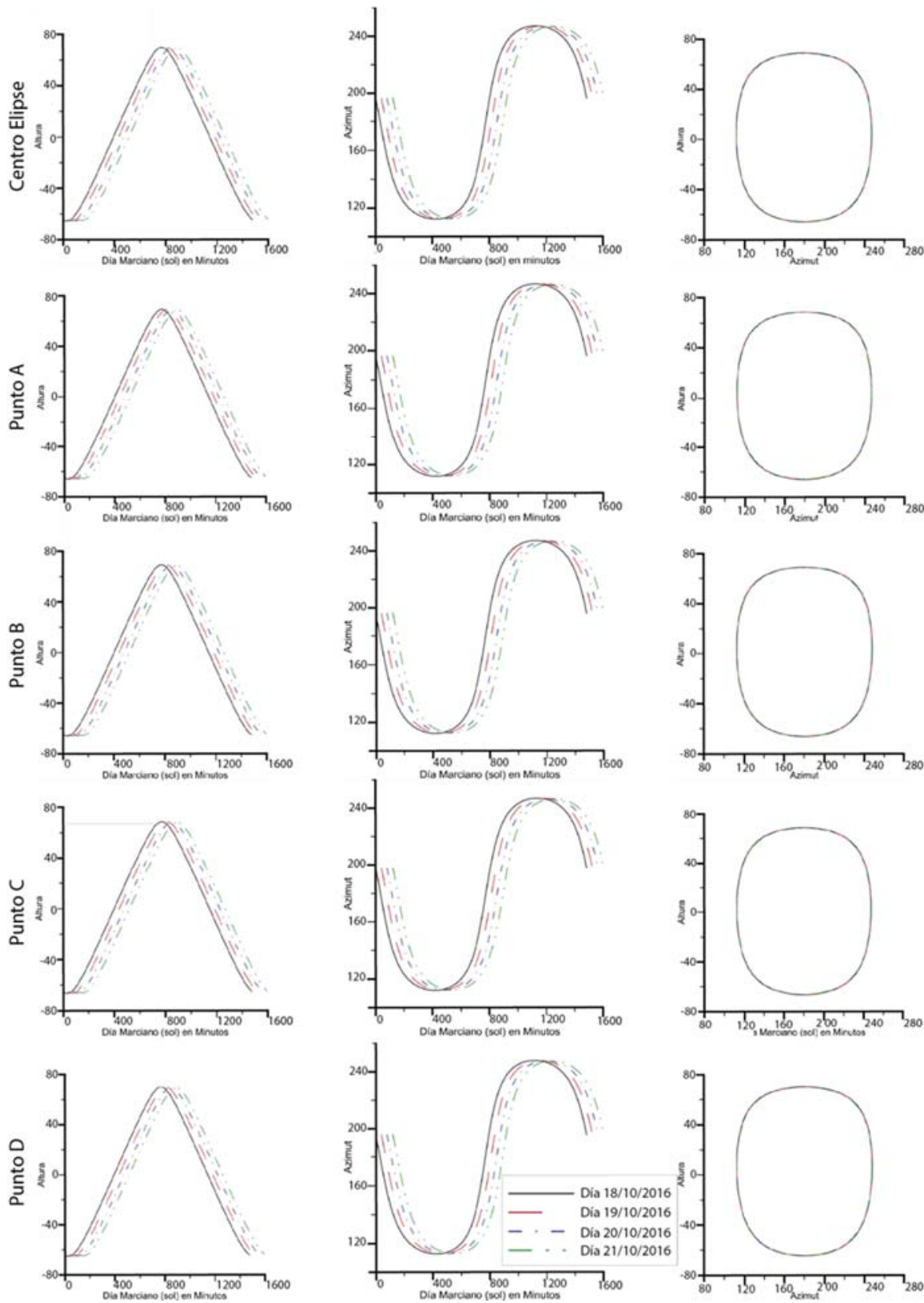

Figura 5. Variaciones de azimut y altura del Sol para el centro y los puntos A, B, C y D de la elipse por sol (18-21/oct/2016). 


\section{Conclusiones}

En este trabajo se ha determinado el cronograma necesario para la observación del Sol mediante el sensor SIS del módulo Schiaparelli sobre la superficie de Marte durante el tiempo previsto de vida de la misión. Debido a la incertidumbre sobre el lugar de amartizaje, para el establecimiento del cronograma se ha realizado una simulación que permita determinar el cronograma de visibilidad del Sol más probable y acotar los márgenes de error. Los cronogramas elaborados incluyen el azimut y la determinación en TU de los instantes de orto y ocaso del Sol.

Los resultados principales a la hora de establecer el cronograma de observación permiten fijar para el centro de la elipse, en cuatro soles que abarcan aproximadamente del 18/oct/2016 al 21/oct/2016, los valores del azimut en el orto variando entre $112^{\circ} .52$ y $112^{\circ} .91$ y en el ocaso variando de $247^{\circ} .40$ a $247^{\circ} .03$. Las máximas diferencias entre los valores obtenidos para el centro y para un punto dentro de la elipse son de $\pm 0^{\circ} .02$ para el azimut del orto y del ocaso. Los instantes de tiempo para el orto en el centro de la elipse varían entre 6:38 TU y 8:38 TU y entre 19:04 TU y 21:05 TU para el ocaso para los cuatro soles. Dependiendo de la latitud de amartizaje final, el tiempo que de media está el Sol por encima del horizonte, de 12 horas 26 minutos, puede variar en 1 minuto. En los 4 soles, para un mismo lugar, la iluminación solar puede asimismo aumentar en 1 minuto.

Finalmente, se ha determinado la correspondencia de ceros entre las escalas de Tiempo Universal terrestre y de Local True Solar Time en el lugar de amartizaje, calculando los valores del TU correspondiente a las $0^{\mathrm{h}}$ de LTST (respecto al meridiano superior), conjuntamente con la altura del Sol correspondiente a la culminación superior.

\section{Agradecimientos}

Esta Investigación ha sido financiada por los proyectos Análisis, modelización y explotación de datos en la exploración de Marte: Misiones EXOMARS2016 y EXOMARS2018 (referencias ESP2016-79135-R y PR26/16-20246)

\section{Referencias}

ÁLVAREZ, M.; HERNANDO, C.; JIMÉNEZ, J.J.; ÁLVAREZ, F.J.; MARTÍN, I.; ESCRIBANO, D. (2014). TID Test Results of Optical Materials and Photodiodes for SIS Instrument (Dreams Project). En Radiation Effects Data Workshop (REDW), IEEE, 1-3, 14-18.

BARDERAS, G., ROMERO, P., VÁZQUEZ, L., VÁZQUEZ-POLETTI, J.L., LLORENTE, I. M. (2012). Opportunities to observe solar eclipses by Phobos with the Mars Science Laboratory. Monthly Notices of the Royal Astronomical Society. 426, 3195-3200. 
BARDERAS, G., ROMERO, P. (2013). Observations of Phobos shadow: Analysis of parameters connecting Earth-Mars reference frames. Planetary and Space Science, 87, 30-36.

BETTANINI, C., ESPÓSITO, F., DEBEI, S., MOLFESE, C., ARRUEGO RODRÍGUEZ, I., COLOMBATTI, G., HARRI, A.M., MONTMESSIN, F., WILSON, C., ABOUDAN, A., ABBAKI, S., APÉSTIGUE, V., BELLUCCI, G., BERTHELIER, J.J., BRUCATO, J., CALCUTT, S., CORTECCHIA, F., DI ACHILLE, G., FERRI, F., FORGET, F., GUIZZO, G., FRISO, E., GENZER, M., GILBERT, P., HAUKKA, H., JIMÉNEZ, J., JIMÉNEZ, S., JOSSET, J.L., KARATEKIN, O., LANDIS, G., LORENZ, R., MARTINEZ, J., MARTY, L., MENNELLA, V., MOHLMANN, D., MOIRIN, D., MOLINARO, R., PALOMBA, E., PATEL, M., POMMEREAU, J.P., POPA, C., RAFKIN, S., RANNOU, P., RENNO, N., SCHIPANI, P., SCHMIDT,W., SILVESTRO, S., SIMOES, F., SPIGA, A., VALERO, F., VÁZQUEZ, L., VIVAT, F.,WITASSE, O., MUGNUOLO, R., PIRROTTA, S., MARCHETTI, E. (2014). The DREAMS experiment on the ExoMars 2016 mission for the study of Martian environment during the dust storm season, en: Metrology for Aerospace (MetroAeroSpace), 2014 IEEE, 167-173.

BURKHART, P.D. (2006). MSL Update to Mars Coordinate Frame Definitions. Interoffice Memorandum 343B-2006-004. JPL.

SILVESTRO, S., VAZ, D.A., DI ACHILLE, G., ESPÓSITO, F., POPA, C. (2014). Eolian characterization of the 2016 ExoMars landing site: implications for the DREAMS (dust characterization, risk assessment and environment analyzer on the Martian surface) experiment. 45th Lunar and Planetary Science Conference, 1887.

ESA ROBOTIC EXPLORATION OF MARS WEBSITE OF THE EUROPEAN SPACE AGENCY (2016). ExoMars Trace Gas Orbiter and Schiaparelli Mission 2016. http:/exploration.esa.int/mars/46124-mission-overview/. Último acceso: 21/jun/2016.

FOLKNER, W.M., WILLIAMS, J.G. (2008). The Planetary and Lunar Ephemeris DE 421. Interoffice Memorandum 343R-08-003. JPL.

GOICOECHEA, S. (2015). Establecimiento de un Cronograma de Observaciones Solares para el sensor SIS en la misión ExoMars16. Trabajo Fin de Grado, supervisado por G. Barderas y P. Romero. Universidad Complutense de Madrid.

MONTENBRUCK, O., Gill, E. (2000). Satellite orbits: Models, methods and applications. Springer-Verlag

PHARR, M., HUMPHREYS, G. (2004). Monte Carlo Integration I: Basic Concepts, en: Physically Based Rendering: From Theory to Implementation. Morgan Kaufmann series in interactive 3D technology. Elsevier Science, 631-660.

PORTIGLIOTTI, S., DUMONTEL, M., CAPUANO, M., LORENZONI, L. (2014). Landing site targeting and constraints for ExoMars 2016 mission, IIPW7.

ROMERO, P., BARDERAS, G., VÁZQUEZ-POLETTI, J.L., LLORENTE, I.M. (2011). Spatial chronogram to detect Phobos eclipses on Mars with the MetNet Precursor Lander. Planetary and Space Science, 59, 1542-1550. 
STANDISH, E.M., WILLIAMS, J.G. (2009). Orbital Ephemerides of the Sun, Moon, and Planets. Informe Técnico. JPL.

VAGO, J., WITASSE, O., SVEDHEM, H., BAGLIONI, P., HALDEMANN, A., GIANFIGLIO, G., BLANCQUAERT, T., MCCOY, D., DE GROOT, R. (2015). ESA ExoMars Program: The next Step in Exploring Mars. Solar System Research, Vol. 49, No. 7, pp. 518-528. 